\title{
Phthalate residue in goat milk-based infant formulas manufactured in China
}

\author{
W. P. Ge,${ }^{*}$ X. J. Yang, ${ }^{*}$ X. Y. Wu, † Z. Wang, $\ddagger$ W. Geng,, and C. F. Guo*1 \\ ${ }^{*}$ College of Food Science and Engineering, Northwest A\&F University, Yangling 712100, China \\ †Xianyang Food and Drug Inspection Center, Xianyang 712000, China \\ ¥Shaanxi Baiyue Youlishi Dairy Co. Ltd., Xianyang 713300, China \\ §Xianyang Bureau of Quality and Technical Supervision, Xianyang 712000, China
}

\begin{abstract}
Phthalates adversely affect the male reproductive system in humans. Through gas chromatography-mass spectrometry analysis, we investigated the residual profile and levels of 15 phthalates in 90 goat milk-based infant formulas from 15 commercial brands of 10 dairy enterprises located in Shaanxi Province, China. In general, dibutyl phthalate was the most detected phthalate, followed by bis(2-ethylhexyl) phthalate, diisobutyl phthalate, and dimethyl phthalate; their geometric mean concentrations in the formulas were 38.1, 24.2, 16.6 , and $8.7 \mu \mathrm{g} / \mathrm{kg}$, respectively. Other phthalates were not detected in the investigated samples. No significant differences were found in the phthalate levels among different stages of infant formulas, even though the samples were packaged in different types of containers. These findings demonstrate that goat milk-based infant formulas may represent the main source of exposure to phthalates in infants.
\end{abstract}

Key words: phthalate, infant formula, goat milk powder

\section{INTRODUCTION}

Diesters of phthalic acid, commonly referred to as phthalates, have been widely used in plastic manufacturing since the 1930s and can be found as a common additive in paints, lubricants, adhesives, insecticides, packaging, and cosmetics (Gao and Wen, 2016). The most important application of phthalates is as plasticizers to improve the flexibility and workability of polymeric materials (Swan, 2008). Phthalates, as plasticizers, are only physically, not chemically, bound to polymer chains, and therefore tend to leach out from polymeric materials and be released into food when polymeric materials containing these compounds are used as packaging (Zhang et al., 2011).

Phthalates have become ubiquitous food contaminants (Cao, 2010). Human exposure to phthalates has

Received February 20, 2016.

Accepted June 26, 2016.

${ }^{1}$ Corresponding author: gcf@nwafu.edu.cn become an important issue because of their alarming effects in newborns, infants, and toddlers, discovered in epidemiological studies. Four studies from Taiwan (Hsu et al., 2012), Sweden (Bornehag et al., 2004), Bulgaria (Kolarik et al., 2008), and the United States (Just et al., 2012) suggest that childhood exposure to bis(2-ethylhexyl) phthalate (DEHP) and butyl benzyl phthalate (BBP) may increase the risk of allergic diseases, including asthma and eczema.

Four prospective cohorts reported that gestational BBP, DEHP, dibutyl phthalate (DBP), and diethyl phthalate (DEP) exposures were associated with alterations in infant or toddler physical development (Engel et al., 2010; Kim et al., 2011; Miodovnik et al., 2011; Whyatt et al., 2012), as well as parent-reported externalizing and internalizing problems (Whyatt et al., 2012) and child behavior similar to autism (Miodovnik et al., 2011). The latest studies reported that fetal and newborn exposure to phthalates causes a decrease in anogenital distance in male newborns (Bornehag et al., 2015; Swan et al., 2015).

Foodstuffs are the major source of phthalate exposure, particularly for the long-chain phthalates (Wittassek et al., 2011). Infants take milk as their main source of nutrition; thus, monitoring the levels of phthalates in formulas is important to provide data for exposure assessment of phthalates in infants. Some studies have reported that cow milk-based infant formulas contain phthalate residue (Mortensen et al., 2005; Yano et al., 2005), but little or no information has been reported on phthalate residue in goat milk-based infant formulas.

China is one of the largest producers of dairy goats and goat milk-based infant formulas in world (Zhao et al., 2011). The objective of the present study was to evaluate the profile and level of phthalate residue in goat milk-based infant formulas manufactured in China.

\section{MATERIALS AND METHODS}

\section{Reagents and Solvents}

Acetonitrile and hexane were HPLC-grade and were obtained from Fisher Scientific (Pittsburgh, PA). The 15 
phthalate standards were dimethyl phthalate (DMP), DEP, diisobutyl phthalate (DIBP), DBP, bis(4-methyl-2-pentyl) phthalate, bis(2-methoxyethyl) phthalate, dipentyl phthalate, bis(2-ethoxyethyl) phthalate, dihexyl phthalate, BBP, bis(2-n-butoxyethyl) phthalate, DEHP, dicyclohexyl phthalate, diphenyl phthalate, and dinonyl phthalate. The phthalate standards were purchased from o2si Smart Solutions (Charleston, SC) in a mixed standard solution with a purity of $>98.0 \%$ for each.

\section{Sample Collection}

Ninety powdered infant formulas were collected from stores of 10 randomly selected dairy enterprises in Shaanxi Province, China. These samples covered 15 commercial brands. For each brand, 2 samples, with plastic bag and metal can packaging, respectively, were collected from stage I (for infants $0-6$ mo old), stage II (for infants 6-12 mo old), and stage III (for infants 12-36 mo old) infant formulas.

\section{Sample Preparation}

Sample preparation was performed according to the method described by Schecter et al. (2013) with minor modification. The samples of powered infant formula (1 g) were directly extracted with $10 \mathrm{~mL}$ of acetone:hexane $(1: 1, \mathrm{vol} / \mathrm{vol}) 3$ times and then centrifuged $(8,000 \times g, 10$ min, room temperature) after shaking for $30 \mathrm{~min}$. The upper organic layers were combined and concentrated to near dryness. The sample residue was redissolved in $2 \mathrm{~mL}$ of hexane and subjected to a glass column packed with $7 \mathrm{~g}$ of Florisil (60-100 mesh, Sigma-Aldrich, St. Louis, MO) conditioned with $20 \mathrm{~mL}$ of acetone:hexane $(2: 8, \mathrm{vol} / \mathrm{vol})$ and $20 \mathrm{~mL}$ of hexane. Phthalates were eluted with $70 \mathrm{~mL}$ of acetone:hexane $(2: 8, \mathrm{vol} / \mathrm{vol})$. The final eluate was concentrated to $5 \mathrm{~mL}$ under a gentle stream of nitrogen for instrumental analysis.

\section{Instrumental Analysis}

The 15 phthalates were measured using a GC (7890A; Agilent Technologies, Santa Clara, CA) coupled with an MS (5975C; Agilent Technologies) equipped with an electron impact ion source in the selective ion monitoring mode. The GC-MS operating conditions were set at $70 \mathrm{eV}$ ionization potential with the source at $230^{\circ} \mathrm{C}$ and electron multiplier voltage at $2,000 \mathrm{eV}$. The injection port was maintained at $250^{\circ} \mathrm{C}$ and $1 \mu \mathrm{L}$ of the sample was injected in splitless mode, followed by a 1-min purge after the injection. Helium was used as the carrier gas at a flow rate of $1.2 \mathrm{~mL} / \mathrm{min}$. A fused-silica capillary column (Rxi-5Sil MS; $30 \mathrm{~m} \times 0.25 \mathrm{~mm}$ i.d. and 0.25 $\mu \mathrm{m}$ film thickness; Restek, Bellefonte, PA) was used for separation. Oven temperature was programmed at $100^{\circ} \mathrm{C}$ (held for $1.0 \mathrm{~min}$ ) and raised to $260^{\circ} \mathrm{C}$ at $8^{\circ} \mathrm{C} /$ min (held for $9 \mathrm{~min}$ ). The retention times on the Rxi5Sil MS column and the fragment ions monitored for the 15 phthalates are listed in Table 1.

\section{Quality Control and Quality Assurance}

Glassware was baked at $450^{\circ} \mathrm{C}$ overnight. The detailed quality-control operations were performed according to the method described by Fierens et al. (2012). The efficiency of the analytical method was evaluated before sample analysis. The limits of detection (LOD) for the 15 phthalates were estimated as $3 \times S_{0}$, where $S_{0}$ is the value of the standard deviation as the concentration approaches zero (Blount et al., 2000). The $S_{0}$ was determined by analyzing quintuplicate sets of the lowest 5 standards and plotting the standard deviation versus the known standard concentration. The y-intercept of the best-fit line of this plot was used as $S_{0}$. Recovery was determined based on the results of 6 replicate analyses of a sample spiked at a $0.10 \mathrm{mg} / \mathrm{kg}$ level of each phthalate standard. The recovery of each phthalate was calculated as the ratio between the experimentally observed concentration and the theoretical concentration as a percentage. Precision was determined from these data by calculating the relative standard deviation of each phthalate.

\section{Data Treatments and Statistics}

Both DBP and DEHP were present in the procedural blank, which was carried through the same processing procedure as the samples, but no samples were used. Their values were subtracted from sample values. Statistical analyses were performed using the statistical software package SPSS (version 15.0, SPSS Inc., Chicago, IL). For concentrations below the LOD, a value equal to the LOD divided by the square root of 2 was used (Hornung and Reed, 1990). The analyses were considered statistically significant when $P<0.05$.

\section{RESULTS AND DISCUSSION}

\section{Method Performance}

To ensure the reliability of the results, instrumental and method performance was evaluated before sample analysis. Table 1 indicates the retention time and the main ions monitored for each phthalate. Confirmation of each phthalate was performed by comparison of the retention time and fragments (abundance ratios between quantitative and qualitative ions) with those 
of standards. The performance characteristics of the method are also summarized in Table 1. For the recovery experiment, the spiked concentration was designed based on the phthalate concentrations detected in the first samples. Each sample for the recovery experiment was also measured without the addition of phthalate compounds to determine the appropriate blank concentration. Recoveries varied between 90 and $102 \%$. The precision, expressed as the relative standard deviation, for each phthalate compound was lower than $10 \%$. The LOD for each phthalate ranged from 5 to $15 \mu \mathrm{g} /$ $\mathrm{kg}$, which was comparable with that of the methods developed by other researchers (Sharman et al., 1994; Sørensen, 2006).

\section{Phthalates Levels in Infant Formulas}

The number of positive samples for each phthalate compound in the investigated formulas is reported in Table 2. The most detected phthalate was DBP, followed by DEHP, DIBP, and DMP. In particular, DBP could be detected in almost every sample. On the contrary, the other 11 phthalates [DEP, bis(4-methyl2-pentyl) phthalate, bis(2-methoxyethyl) phthalate, dipentyl phthalate, bis(2-ethoxyethyl) phthalate, dihexyl phthalate, BBP, bis(2-n-butoxyethyl) phthalate, dicyclohexyl phthalate, diphenyl phthalate, and DNOP] were not detected in any of the samples. This finding differed from the results of the Ministry of Agriculture, Fisheries, and Food (1996), which reported that BBP was also detected in infant formulas at a level of $<0.004$ to $0.25 \mathrm{mg} / \mathrm{kg}$. One possible explanation for the dis- crepancy in the results is that China prohibits the use of BBP as plasticizers of food containers and packaging materials, whereas the United Kingdom permits the use of $\mathrm{BBP}$ in food containers and packaging materials (Jobling et al., 1995). The phthalates detected in our study, namely DBP, DEHP, DIBP, and DMP, are permitted for use as plasticizers in food containers and packaging materials in China, which suggests that the phthalates detected in the infant formulas directly or indirectly came from plastic materials.

Phthalate concentrations observed in the different groups of infant formulas are also summarized in Table 2. In general, DBP levels were the highest of all phthalate levels in every investigated group, followed by DEHP, DIBP, and DMP. This finding differed from the phthalate residual profile in cow milk-based infant formulas, where DEHP was the most abundant phthalate compound (Yano et al., 2005; Sørensen, 2006). Maximum concentrations of DBP and DEHP were detected in formula stages II and III respectively, whereas maximum concentrations of DMP and DIBP were detected in all stage I formulas. According to the Chinese hygienic standards for uses of additives in food containers and packaging materials (Ministry of Health of the People's Republic of China and Standardization Administration of the People's Republic of China, 2008), the specific migration limits (maximum allowed concentrations) in foods for DBP and DEHP were 0.3 and $1.5 \mathrm{mg} / \mathrm{kg}$, respectively. The DBP and DEHP levels of all tested samples were not beyond the set limits, except 1 sample of stage II infant formula whose DBP level exceeded the limit value over 1.7 times. The

Table 1. Summary of analytical method ${ }^{1}$

\begin{tabular}{|c|c|c|c|c|c|c|c|}
\hline Phthalate $^{2}$ & CAS No. & $\begin{array}{l}\text { Retention time } \\
\text { (min) }\end{array}$ & $\begin{array}{l}\text { Quantifier ion } \\
\qquad(m / z)\end{array}$ & $\begin{array}{l}\text { Qualifier ion } \\
\qquad(m / z)\end{array}$ & $\begin{array}{l}\text { LOD } \\
(\mu \mathrm{g} / \mathrm{kg})\end{array}$ & $\begin{array}{c}\text { Recovery } \\
(\%)\end{array}$ & $\begin{array}{c}\mathrm{RSD} \\
(\%)\end{array}$ \\
\hline $\mathrm{DMP}$ & $131-11-3$ & 8.84 & 163 & 135,194 & 8 & 91 & 4 \\
\hline DEP & $84-66-2$ & 11.02 & 149 & 177,121 & 5 & 90 & 6 \\
\hline $\mathrm{DBP}$ & $84-74-2$ & 16.20 & 149 & 223,205 & 5 & 94 & 7 \\
\hline BMPP & $117-82-8$ & 16.64 & 149 & 104,207 & 8 & 100 & 8 \\
\hline DMEP & $146-50-9$ & $17.57\left(17.62^{3}\right)$ & 149 & 167,251 & 8 & 93 & 6 \\
\hline $\mathrm{BBP}$ & $85-68-7$ & 20.88 & 149 & 206,123 & 5 & 98 & 9 \\
\hline DBEP & $117-83-9$ & 22.23 & 149 & 101,193 & 15 & 90 & 5 \\
\hline DEHP & $84-61-7$ & 22.98 & 149 & 167,249 & 8 & 93 & 7 \\
\hline DCHP & $117-81-7$ & 23.14 & 149 & 167,279 & 6 & 90 & 6 \\
\hline DPHP & $84-62-8$ & 23.34 & 225 & 153,197 & 9 & 93 & 7 \\
\hline DNP & $117-84-0$ & 26.49 & 149 & 279,261 & 10 & 97 & 8 \\
\hline
\end{tabular}

${ }^{1} \mathrm{LOD}=$ limit of detection; RSD $=$ relative standard deviation; $\mathrm{CAS}=$ Chemical Abstracts Service.

${ }^{2} \mathrm{DMP}=$ dimethyl phthalate; $\mathrm{DEP}=$ diethyl phthalate; $\mathrm{DIBP}=$ diisobutyl phthalate; $\mathrm{DBP}=$ dibutyl phthalate; $\mathrm{BMPP}=$ bis $(4-\mathrm{methyl}-2$-pentyl) phthalate; DMEP = bis(2-methoxyethyl) phthalate; DPP = dipentyl phthalate; $\mathrm{DEEP}=\operatorname{bis}(2$-ethoxyethyl $)$ phthalate; $\mathrm{DHXP}=$ dihexyl phthalate; $\mathrm{BBP}=$ butylbenzyl phthalate; DBEP = bis (2-n-butoxyethyl) phthalate; DEHP = bis (2-ethylhexyl) phthalate; DCHP = dicyclohexyl phthalate; DPHP = diphenyl phthalate; DNP = dinonyl phthalate.

${ }^{3}$ Retention time of another isomer. 


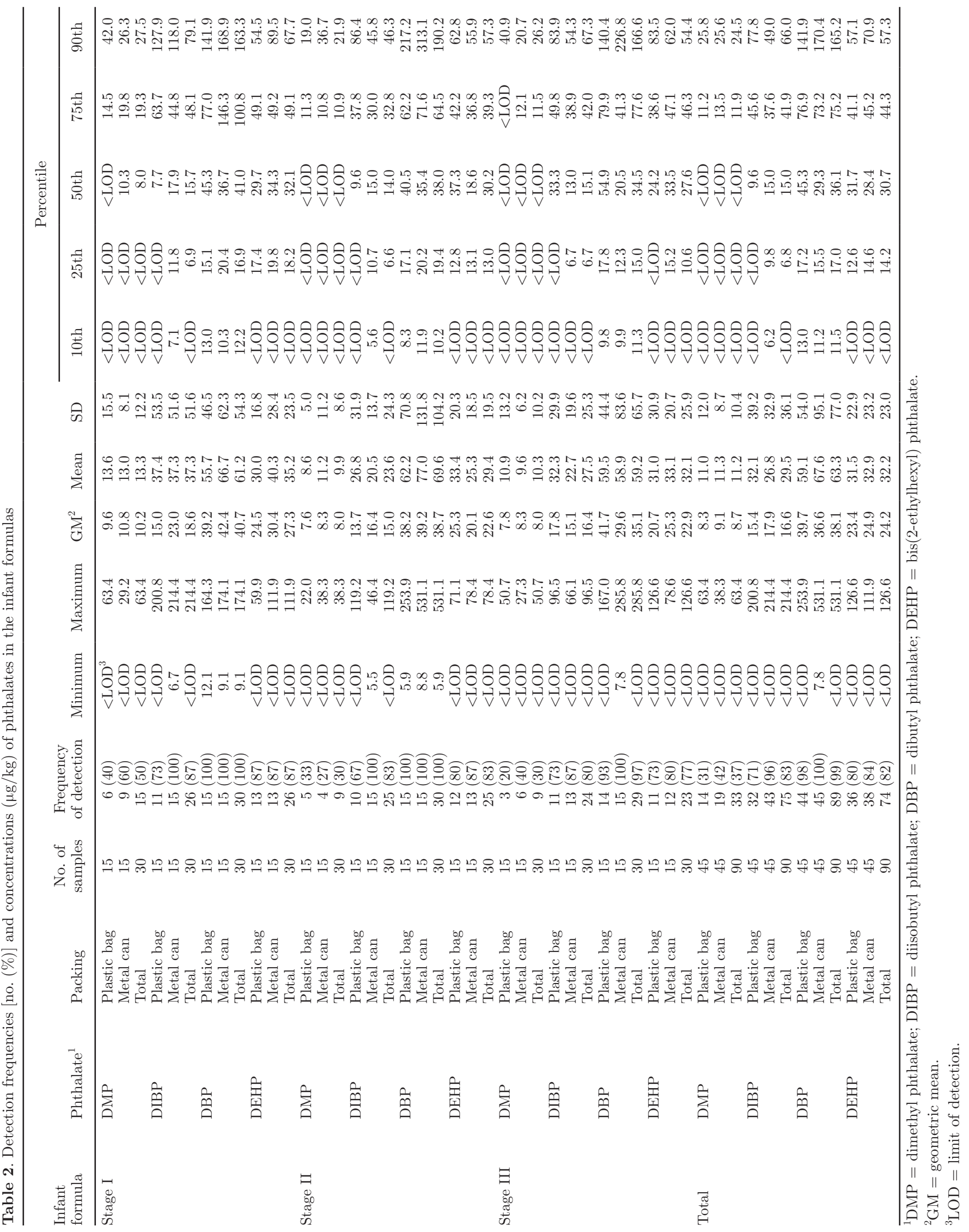


phthalate levels found in our study are comparable with those published by Yano et al. (2005), who collected 27 samples of cow milk-based powdered infant formulas from different countries and analyzed these for DBP and DEHP. They found that DBP and DEHP levels ranged from 15 to 77 and 34 to $281 \mu \mathrm{g} / \mathrm{kg}$, respectively. However, the phthalate levels found in the present study were markedly lower than those reported by Cirillo et al. (2015), who collected 28 samples of powered infant formulas from Italy and also analyzed these for DBP and DEHP. Those authors found that DBP and DEHP levels ranged from 101 to 812 and 702 to $3,552 \mu \mathrm{g} / \mathrm{kg}$, respectively. It is difficult to clearly explain why the levels differ between the studies, because any one of the ingredient sources, manufacturing processes, packing materials, and storage conditions may affect the final concentrations of phthalates in infant formulas.

Data relevant to the detectable phthalate compounds showed wide variability, but no significant $(P>0.05)$ differences were found in the phthalate levels between samples packed with different materials. This differed with the findings of Lin et al. (2015), who reported that the amounts of phthalates in commercial whole milk products packaged in metal containers were much lower than those in plastic containers. The present finding suggests that DBP, DEHP, DIBP, and DMP contamination could come from raw materials and manufacturing processes rather than from the packaging. Phthalates are lipophilic and, therefore, tend to concentrate in the lipid phase of foodstuffs. Goat milk has higher fat content than cow milk (Ceballos et al., 2009); therefore, the former is expected to be more contaminated with phthalates than the latter. Another study (W. P. Ge, unpublished data), which was conducted in Shaanxi Province, China, showed that the phthalate concentrations in raw goat milk are markedly higher than in raw cow milk. Milk is easily contaminated by phthalates during the production or preparation of formulas because of the migration of phthalates from plastic materials in contact with milk (Fierens et al., 2013).

Our recent survey showed that nearly no plastic tubing or containers were used in the dairy factories where we collected the infant formula samples; however, polyvinyl chloride tubing is present in some parts of the milking machines used by dairy farmers. The migration of phthalates from the polyvinyl chloride tubing of the milking machine into raw cow milk during milking at farms was investigated by Castle et al. (1990). Those authors reported that, in controlled milk samples obtained by hand milking, DEHP concentration was below $5 \mu \mathrm{g} / \mathrm{kg}$, whereas, for samples obtained by machine milking, the concentration in the milking chamber for each individual cow averaged $30 \mu \mathrm{g} / \mathrm{kg}$ and rose to 50 $\mu \mathrm{g} / \mathrm{kg}$ in the central collecting tank.
In addition to the goat milk powder, the most commonly used goat milk-based infant formulas contain purified whey as a protein source, a blend of vegetable oils as a fat source, lactose or sucrose as a carbohydrate source, a vitamin-mineral mix, and other ingredients depending on the manufacturer. Goat milk powder is the dominating ingredient in goat milk-based infant formula. The content of goat milk powder in stage III infant formula (50-60\%) was markedly higher than in stage I and stage II (30-35\%). However, we found no significant $(P>0.05)$ differences in the levels of each kind of phthalate between various stage infant formulas. This finding suggests that, in addition to the goat milk powder, the other ingredients should have influenced the levels of phthalate in each stage infant formula. In particular, it has been reported that vegetable oil is easier to be contaminated by phthalates (Xu et al., 2010; Nanni et al., 2011).

\section{CONCLUSIONS}

Our data indicate the presence of DBP, DEHP, DIBP, and DMP in a large number of goat milk-based infant formulas manufactured in China. Our findings demonstrate that goat milk-based infant formulas may be the main source of exposure to phthalates in babies. To protect infants' health, it would be necessary for dairy enterprises to reduce phthalate residues in the products by improving quality management and control of raw materials and milking machines.

\section{ACKNOWLEDGMENTS}

This work was supported by the Shaanxi Province science and technology overall planning and innovation project (China; No. 2015KTCQ03-08), the National Natural Science Foundation of China (NSFC; No. 31301444), the Natural Science Foundation of Shaanxi Province, China (No. 2015JM3100), and the Fundamental Research Funds for the Central Universities (China; No. QN2013059).

\section{REFERENCES}

Blount, B. C., K. E. Milgram, M. J. Silva, N. A. Malek, J. A. Reidy, L. L. Needham, and J. W. Brock. 2000. Quantitative detection of eight phthalate metabolites in human urine using HPLC-APCIMS/MS. Anal. Chem. 72:4127-4134.

Bornehag, C. G., F. Carlstedt, B. A. G. Jonsson, C. H. Lindh, T. K. Jensen, A. Bodin, C. Jonsson, S. Janson, and S. H. Swan. 2015. Prenatal phthalate exposures and anogenital distance in Swedish boys. Environ. Health Perspect. 123:101-107.

Bornehag, C. G., J. Sundell, C. J. Weschler, T. Sigsgaard, B. Lundgren, M. Hasselgren, and L. Hagerhed-Engman. 2004. The association between asthma and allergic symptoms in children and phthalates in house dust: A nested case-control study. Environ. Health Perspect. 112:1393-1397. 
Cao, X. L. 2010. Phthalate esters in foods: Sources, occurrence, and analytical methods. Compr. Rev. Food Sci. Food Saf. 9:21-43.

Castle, L., J. Gilbert, and T. Eklund. 1990. Migration of plasticizer from poly(vinyl chloride) milk tubing. Food Addit. Contam. 7:591-596.

Ceballos, L. S., E. R. Morales, G. D. T. Adarve, J. D. Castro, L. P. Martinez, and M. R. S. Sampelayo. 2009. Composition of goat and cow milk produced under similar conditions and analyzed by identical methodology. J. Food Compos. Anal. 22:322-329.

Cirillo, T., G. Latini, M. A. Castaldi, L. Dipaola, E. Fasano, F. Esposito, G. Scognamiglio, F. Di Francesco, and L. Cobellis. 2015. Exposure to di-2-ethylhexyl phthalate, di-n-butyl phthalate and bisphenol A through infant formulas. J. Agric. Food Chem. 63:3303-3310.

Engel, S. M., A. Miodovnik, R. L. Canfield, C. B. Zhu, M. J. Silva, A. M. Calafat, and M. S. Wolff. 2010. Prenatal phthalate exposure is associated with childhood behavior and executive functioning. Environ. Health Perspect. 118:565-571.

Fierens, T., K. Servaes, M. Van Holderbeke, L. Geerts, S. De Henauw, I. Sioen, and G. Vanermen. 2012. Analysis of phthalates in food products and packaging materials sold on the Belgian market. Food Chem. Toxicol. 50:2575-2583

Fierens, T., M. Van Holderbeke, H. Willems, S. De Henauw, and I. Sioen. 2013. Transfer of eight phthalates through the milk chainA case study. Environ. Int. 51:1-7.

Gao, D.-W., and Z.-D. Wen. 2016. Phthalate esters in the environment: A critical review of their occurrence, biodegradation, and removal during wastewater treatment processes. Sci. Total Environ. 541:986-1001.

Hornung, R. W., and L. D. Reed. 1990. Estimation of average concentration in the presence of nondetectable values. Appl. Occup. Environ. Hyg. 5:46-51.

Hsu, N. Y., C. C. Lee, J. Y. Wang, Y. C. Li, H. W. Chang, C. Y. Chen, C. G. Bornehag, P. C. Wu, J. Sundell, and H. J. Su. 2012. Predicted risk of childhood allergy, asthma, and reported symptoms using measured phthalate exposure in dust and urine. Indoor Air 22:186-199.

Jobling, S., T. Reynolds, R. White, M. G. Parker, and J. P. Sumpter. 1995. A variety of environmentally persistent chemicals, including some phthalate plasticizers, are weakly estrogenic. Environ. Health Perspect. 103:582-587.

Just, A. C., R. M. Whyatt, M. S. Perzanowski, A. M. Calafat, F. P. Perera, I. F. Goldstein, Q. X. Chen, A. G. Rundle, and R. L. Miller. 2012. Prenatal exposure to butylbenzyl phthalate and early eczema in an urban cohort. Environ. Health Perspect. 120:14751480.

Kim, Y., E. H. Ha, E. J. Kim, H. Park, M. Ha, J. H. Kim, Y. C. Hong, N. Chang, and B. N. Kim. 2011. Prenatal exposure to phthalates and infant development at 6 months: Prospective mothers and children's environmental health (MOCEH) study. Environ. Health Perspect. 119:1495-1500.

Kolarik, B., K. Naydenov, M. Larsson, C. G. Bornehag, and J. Sundell. 2008. The association between phthalates in dust and allergic diseases among Bulgarian children. Environ. Health Perspect. 116:98-103.

Lin, J., W. Chen, H. Zhu, and C. Wang. 2015. Determination of free and total phthalates in commercial whole milk products in different packaging materials by gas chromatography-mass spectrometry. J. Dairy Sci. 98:8278-8284.
Ministry of Agriculture, Fisheries, and Food. 1996. Phthalates in infant formulae. Food surveillance information sheet, No. 83. Ministry of Agriculture, Fisheries, and Food, London, UK.

Ministry of Health of the People's Republic of China, and Standardization Administration of the People's Republic of China. 2008 GB 9685-2008. Hygienic standards for uses of additives in food containers and packaging materials. Beijing, China.

Miodovnik, A., S. M. Engel, C. B. Zhu, X. Y. Ye, L. V. Soorya, M. J. Silva, A. M. Calafat, and M. S. Wolff. 2011. Endocrine disruptors and childhood social impairment. Neurotoxicology 32:261-267.

Mortensen, G. K., K. M. Main, A. M. Andersson, H. Leffers, and N. E. Skakkebwk. 2005. Determination of phthalate monoesters in human milk, consumer milk, and infant formula by tandem mass spectrometry (LC-MS-MS). Anal. Bioanal. Chem. 382:1084-1092.

Nanni, N., K. Fiselier, K. Grob, M. Di Pasquale, L. Fabrizi, P. Aureli, and E. Coni. 2011. Contamination of vegetable oils marketed in Italy by phthalic acid esters. Food Contr. 22:209-214.

Schecter, A., M. Lorber, Y. Guo, Q. Wu, S. H. Yun, K. Kannan, M. Hommel, N. Imran, L. S. Hynan, D. L. Cheng, J. A. Colacino, and L. S. Birnbaum. 2013. Phthalate concentrations and dietary exposure from food purchased in New York State. Environ. Health Perspect. 121:473-494.

Sharman, M., W. A. Read, L. Castle, and J. Gilbert. 1994. Levels of di-(2-ethylhexyl) phthalate and total phthalate-esters in milk, cream, butter and cheese. Food Addit. Contam. 11:375-385.

Sørensen, L. K. 2006. Determination of phthalates in milk and milk products by liquid chromatography/tandem mass spectrometry. Rapid Commun. Mass Spectrom. 20:1135-1143.

Swan, S. H. 2008. Environmental phthalate exposure in relation to reproductive outcomes and other health endpoints in humans. Environ. Res. 108:177-184.

Swan, S. H., S. Sathyanarayana, E. S. Barrett, S. Janssen, F. Liu, R. H. N. Nguyen, J. B. Redmon, and T. S. Team. 2015. First trimester phthalate exposure and anogenital distance in newborns. Hum. Reprod. 30:963-972.

Whyatt, R. M., X. H. Liu, V. A. Rauh, A. M. Calafat, A. C. Just, L. Hoepner, D. Diaz, J. Quinn, J. Adibi, F. P. Perera, and P. FactorLitvak. 2012. Maternal prenatal urinary phthalate metabolite concentrations and child mental, psychomotor, and behavioral development at 3 years of age. Environ. Health Perspect. 120:290-295.

Wittassek, M., H. M. Koch, J. Angerer, and T. Bruning. 2011. Assessing exposure to phthalates-The human biomonitoring approach. Mol. Nutr. Food Res. 55:7-31.

Xu, Q., X. Y. Yin, M. Wang, H. F. Wang, N. P. Zhang, Y. Y. Shen, S. Xu, L. Zhang, and Z. Z. Gu. 2010. Analysis of phthalate migration from plastic containers to packaged cooking oil and mineral water. J. Agric. Food Chem. 58:11311-11317.

Yano, K., N. Hirosawa, Y. Sakamoto, H. Katayama, T. Moriguchi, and K. Asaoka. 2005. Phthalate levels in baby milk powders sold in several countries. Bull. Environ. Contam. Toxicol. 74:373-379.

Zhang, H., X. Q. Chen, and X. Y. Jiang. 2011. Determination of phthalate esters in water samples by ionic liquid cold-induced aggregation dispersive liquid-liquid microextraction coupled with high-performance liquid chromatography. Anal. Chim. Acta 689:137-142.

Zhao, G. H., M. T. Zhang, L. H. Lei, C. C. Shang, D. Y. Cao, T. T. Tian, J. Li, J. Y. Xu, Y. L. Yao, D. K. Chen, and X. Q. Zhu. 2011. Seroprevalence of Toxoplasma gondii infection in dairy goats in Shaanxi Province, Northwestern China. Parasit. Vectors 4:47. 